\title{
Effects of Wearing Compression Stockings on Exercise Performance and Associated Indicators: A Systematic Review
}

This article was published in the following Dove Press journal: Open Access Journal of Sports Medicine

\author{
Gustavo R Mota $\mathbb{D}^{1}$ \\ Mário Antônio de Moura \\ Simim id ${ }^{2}$ \\ Izabela Aparecida dos Santos' \\ Jeffer Eidi Sasaki (D) \\ Moacir Marocolo (iD ${ }^{3}$ \\ 'Human Performance and Sport \\ Research Group, Department of Sport \\ Sciences, Institute of Health Sciences, \\ Federal University of Triangulo Mineiro, \\ Uberaba, MG, Brazil; ${ }^{2}$ Research Group in \\ Biodynamic Human Movement, Institute \\ of Physical Education and Sports, Federal \\ University of Ceará, Fortaleza, CE, Brazil; \\ ${ }^{3}$ Physiology and Human Performance \\ Research Group, Department of \\ Physiology, Federal University of Juiz de \\ Fora, Juiz de Fora, MG, Brazil
}

\begin{abstract}
This systematic review investigated the effects of wearing below-knee compression stockings (CS) on exercise performance (or sports activity) and associated physiological and perceived indicators. We searched articles on PubMed using the following terms: "graduated compression stockings"; "compression stockings"; "graduated compression socks"; "compression socks" combined with "performance", "athletes", "exercise", "exercise performance", "fatigue", "sports" and "recovery", resulting in 1067 papers. After checking for inclusion criteria (e.g., original studies, healthy subjects, performance analysis), 21 studies were selected and analyzed. We conclude that wearing CS during exercise improved performance in a small number of studies. However, wearing CS could benefit muscle function indicators and perceived muscle soreness during the recovery period. Future research should investigate the chronic effect of CS on Sports Medicine and athletic performance.
\end{abstract}

Keywords: ergogenic aid, fatigue, sports, medicine, prevention, soccer, running

\section{Introduction}

The prevention of deep venous thrombosis is one of the first evidence-based benefits of wearing compression stockings (CS), demonstrated by a clinical experiment in which CS improved the venous return by increasing femoral vein blood flow velocity in hospitalized patients. ${ }^{1}$ Over time, the interest from the basic medical area has expanded to other fields like Sports Medicine. ${ }^{2}$ Nowadays, recreational and professional athletes have used CS as a tool for improving performance or accelerate recovery from training or competitions, and also to reduce lower limb volume, ${ }^{3,4}$ relieve symptoms of muscle soreness, and fatigue. ${ }^{3-6}$ Such popularity is probably boosted by the possibility to obtain potential ergogenic benefits with a simple and low-cost aid.

There are different types (e.g., shorts for thighs, full-leg) and application modes (e.g., using only after the exercise) for compression garments. However, using CS (bellow-knee) "only during" the exercise are probably more practical (than during recovery, after-exercise) for a significant number of sports/activities. For example, uniform issues would limit whole-body garments in some sports. Also, athletes living in tropical locations could be unmotivated to wear compression garments after training sessions once those garments usually promote higher skin temperatures. ${ }^{7,8}$ Additionally, there is limited evidence regarding the effects of wearing CS (only)
Correspondence: Gustavo R Mota Human Performance and Sport Research Group, Department of Sport Sciences/ Institute of Health Sciences, Federal University of Triângulo Mineiro, Av. Tutunas, 490 Uberaba/MG, Uberaba $3806 \mid-500$, Brazil

Tel +55343700-6633

Email grmotta@gmail.com 
during exercise/training/competition, which could be relevant for Sports Medicine professionals. Therefore, the purpose of this systematic review was to investigate the effect of wearing below-knee CS during exercise (or sports activity) on performance and associated physiological and perceptual indicators.

\section{Methods}

A systematic literature search was performed by two independent reviewers in PubMed. The following terms: (i) "graduated compression stockings"; (ii) "compression stockings"; (iii) "graduated compression socks"; (iv) "compression socks" were combined with "performance", "athletes", "exercise", "exercise performance", "fatigue", "sports" and "recovery" (Figure 1).

\section{Inclusion Criteria}

The studies included in this review met the following inclusion criteria: 1) original studies; 2) comprised samples of adults ( $\geq 18 \mathrm{yr}$ ); 3) participants were healthy; 4) investigated the effects of wearing foot-to-knee (below knee) CS (during exercise) on exercise performance and physiological and perceptual indicators (e.g., muscle fatigue, muscle recovery, musle soreness); 5) compression stockings worn during the exercise/test/match; and 6) study protocol included exercise or effort tests and performance analysis.

The literature search occurred between January 01, 1900, until June 30, 2019. We excluded the following type of articles: conference abstracts, case reports, short communications, systematic reviews, meta-analyses, theses, letters to the editor, and protocol papers. Also, we excluded studies involving unhealthy participants: e.g., patients with morbid conditions such as obesity, chronic venous insufficiency, diabetes, hypertension (but not limited to).

\section{Analysis}

The heterogeneity of the selected studies was considerable: e.g., exercise protocols, fitness level of the participants, variables measured. Thus, we have decided not to evaluate the studies chosen from a statistical point of view. Instead, we performed a qualitative analysis, conducted by two authors focusing on the effects reported by the authors and potential practical implications. All other authors read this qualitative analysis carefully, and edits have been incorporated.

\section{Results}

Figure 1 shows the search, selection, and inclusion process. The search displayed a total of 1067 papers, which were reduced to 370 after exclusion of duplicate publications. Then, we discarded 39 articles written in nonEnglish languages. ${ }^{9}$ From the remaining 331 items, we excluded 261 by examining the title. Finally, from the remaining 70 articles, we selected 21 studies for this review according to our inclusion criteria (Figure 1).

Table 1 presents a summary of the studies examining the effects of wearing below-knee CS during exercise on performance and associated indicators. Running was the most common type of exercise in the selected studies ( $76 \%, 16$ out of the 21 studies), followed by soccer (two studies; $10 \%)$, triathlon, calf-rise exercise and cycle ergometer (one study each one; $5 \%$ ). All studies were performed using a randomized experimental design, with the majority employing a crossover design strategy (13 studies, 62\%) (Table 1).

Table 2 presents those studies in which CS influenced at least one measurable variable (15 studies, $71 \%$ ). Three studies (14\%) found effects from wearing CS on at least two variables, and for all others (12 studies; 57\%) CS affected only one variable (Table 2).

Only two studies found some beneficial effect of CS on performance, and a third study improved subsequent performance (Table 2). Two studies did not find performance effects of CS for the group mean, but the authors highlighted that CS promoted benefits for some individuals. The main effects of CS are presented with compressions between 20 and $30 \mathrm{mmHg}$. The range between the minimum compression values is 12 to $28 \mathrm{mmHg}$, while the maximum values range from 15 to $33 \mathrm{mmHg}$.

\section{Discussion}

This systematic review aimed to investigate the effect of wearing below-knee CS during exercise on performance and associated indicators. The main finding is that wearing this kind of CS during exercise (or physical activity) improved performance in a minor part of the studies selected (i.e., 3 out of 21). However, a reasonable number of studies have shown evidence that wearing CS could benefit muscle function or fatigue indicators (e.g., CMJ, specific physical tests) and perceived muscle soreness just after the exercise protocol and/or hours after the exercise bout (e.g., during $1 \mathrm{~h}, 24 \mathrm{~h}$ recovery). 


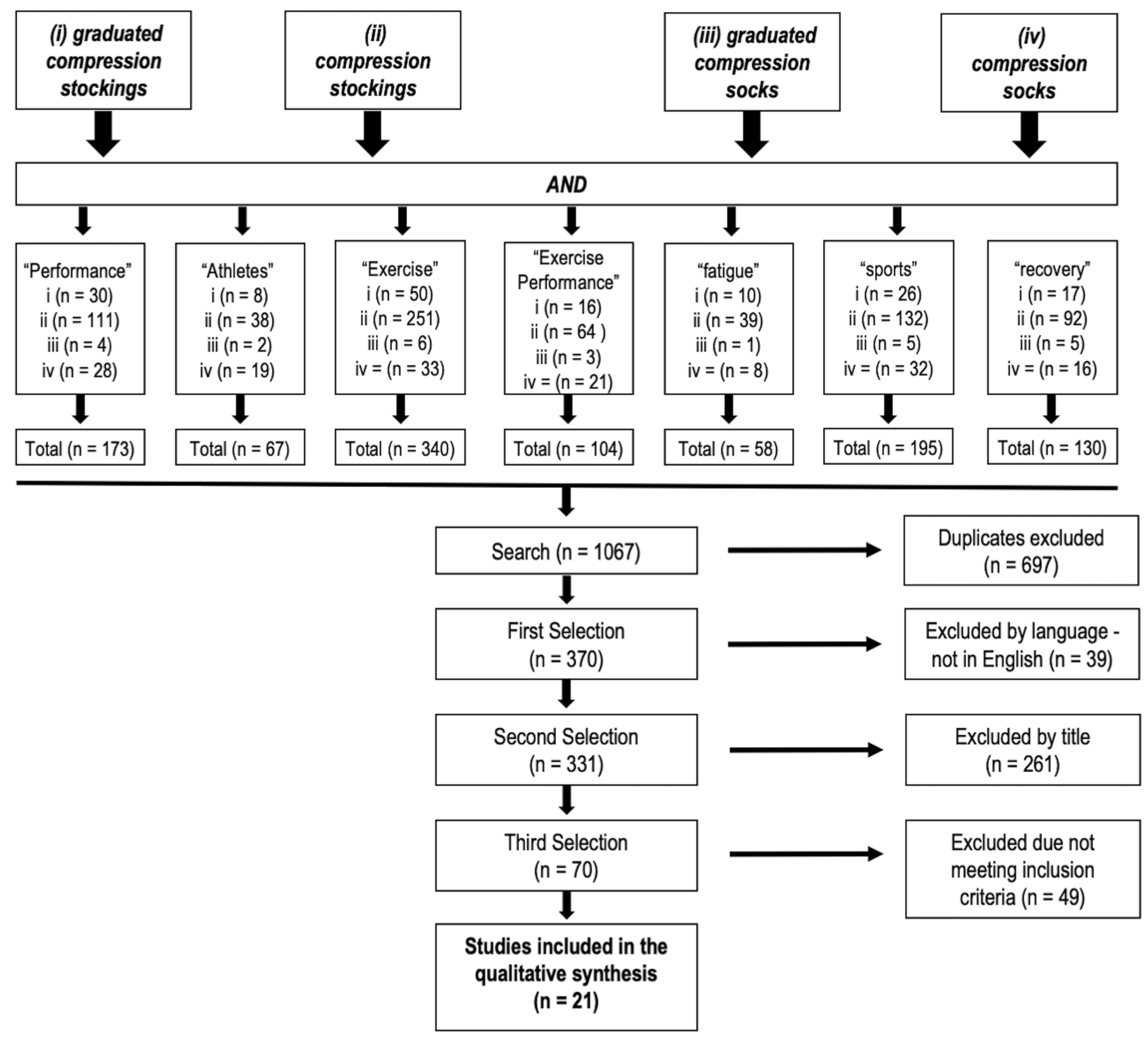

Figure I Flow chart for search and selection of articles.

\section{CS and Performance Improvement}

One of the main reasons for wearing CS during exercise is probably the expectation of performance enhancement due to potential physiological effects. ${ }^{2}$ This includes better venous return which hasten metabolic removal from the exercising muscles ${ }^{31}$ and reduce cardiac load, ${ }^{26}$ improved proprioceptive feedback and better movement accuracy, ${ }^{32}$ reduced muscle oscillations, lower muscle damage, inflammation, and soreness. ${ }^{6,31}$ In the current review, only three studies found some CS-induced benefit on performance but did not present adirect mechanistic explanation. For example, astudy concluded that wearing CS (during two soccer matches,
72 hin-between) resulted in higher distances covered in high-intensity activities which are decisive for soccer. Also, CS promoted alower perceived muscle soreness in thesecond match. ${ }^{17}$ Although the authors did not measure any direct muscle damage marker, they suggested that CS probably protected the eccentric actions common in soccer matches, ${ }^{33}$ mechanically (i.e., smaller muscle oscillation). ${ }^{6}$ In this regard, the oscillating forces experienced by the muscle resulted in reduced muscle fatigue. Thus, the CS might offer a mechanical advantage reducing muscle oscillation and countering fatigue in high-intensity activities (e.g., intermittent acceleration, changing directions). ${ }^{34,35}$ 


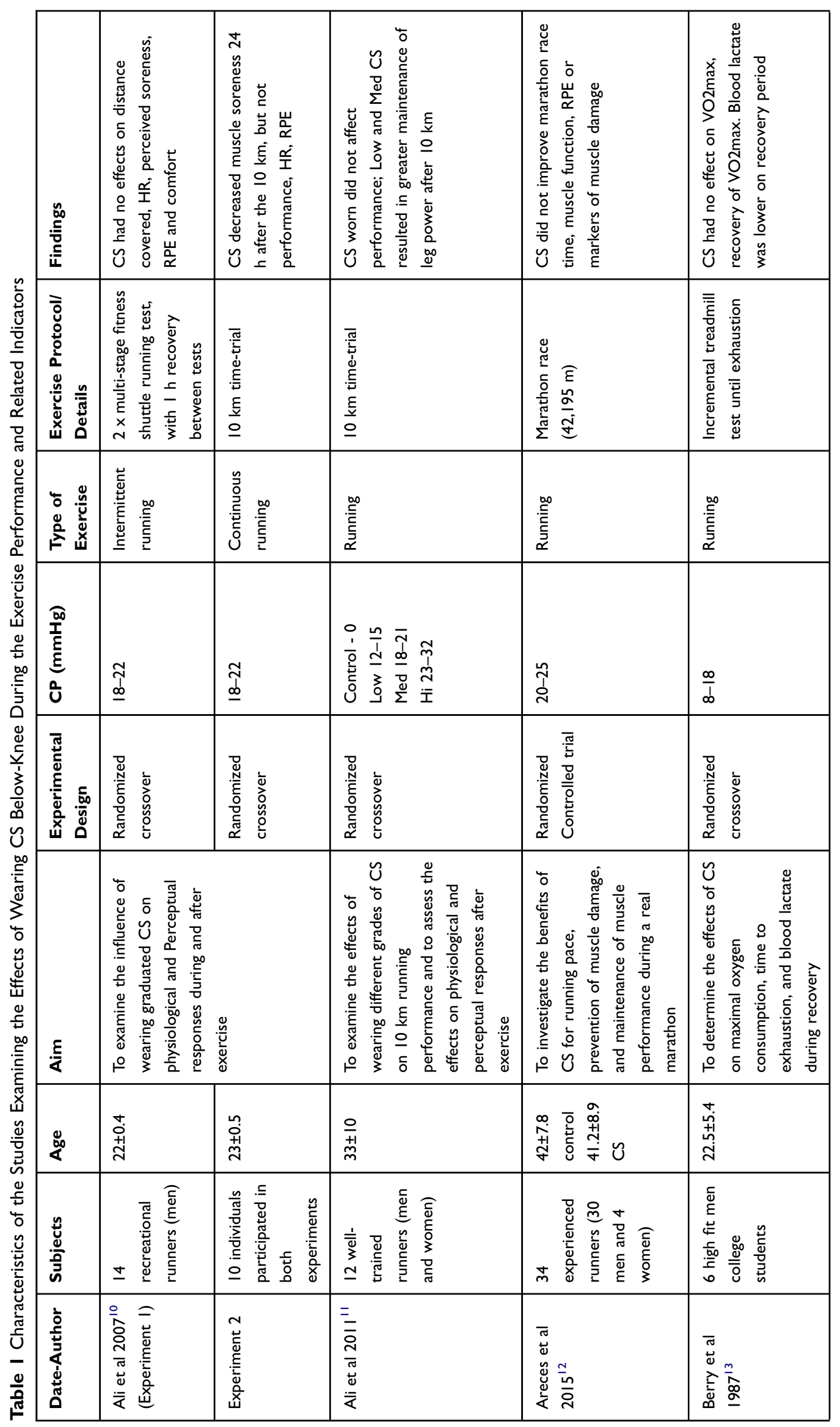




\begin{tabular}{|c|c|c|c|}
\hline 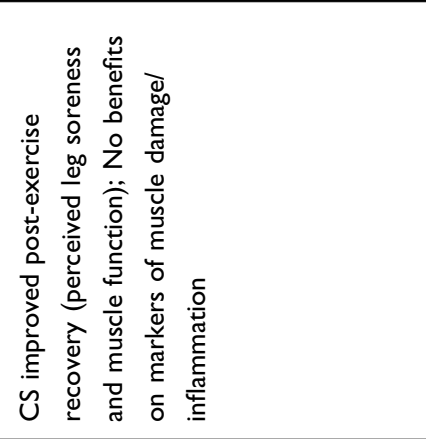 & 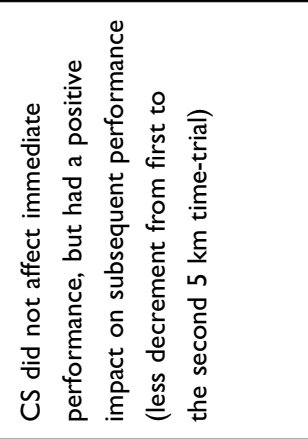 & 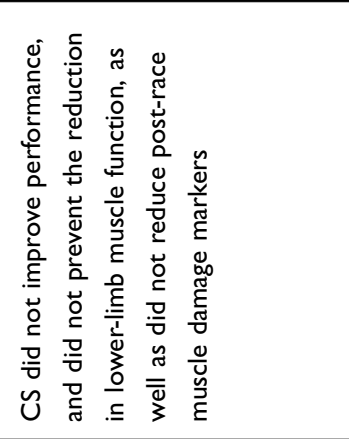 & 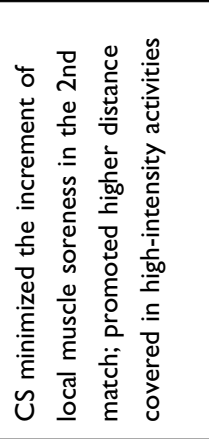 \\
\hline 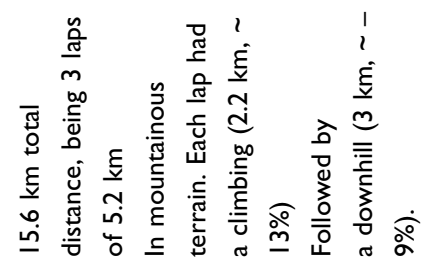 & 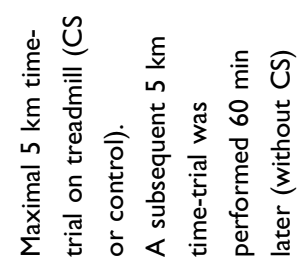 & 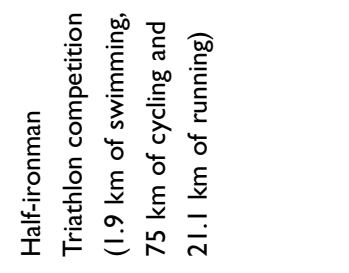 & 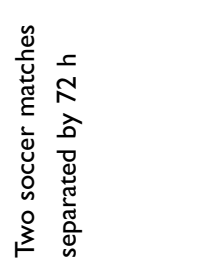 \\
\hline 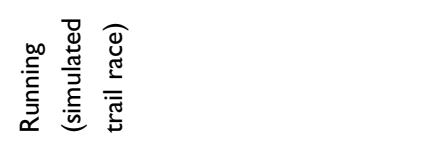 & 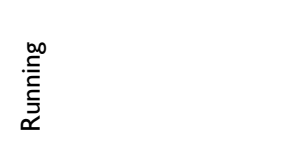 & 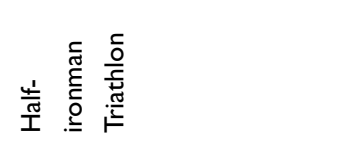 & 这 \\
\hline$\stackrel{\stackrel{\sim}{\sim}}{ }$ & 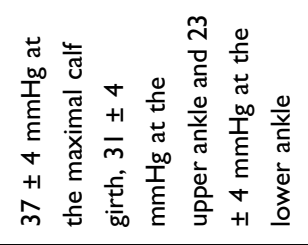 & 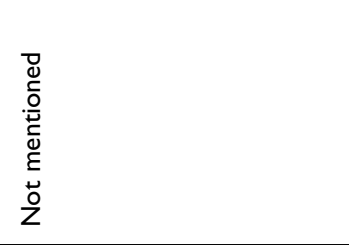 & $\begin{array}{l}\text { ㅇ } \\
\text { ஸे }\end{array}$ \\
\hline 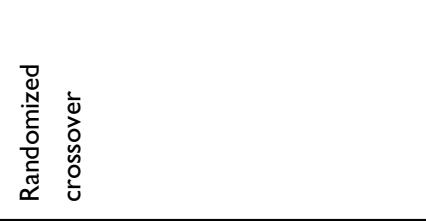 & 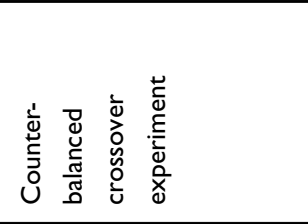 & 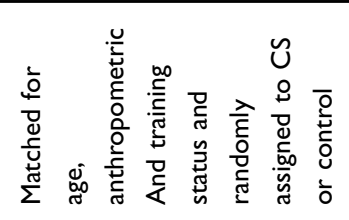 & 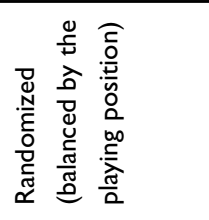 \\
\hline 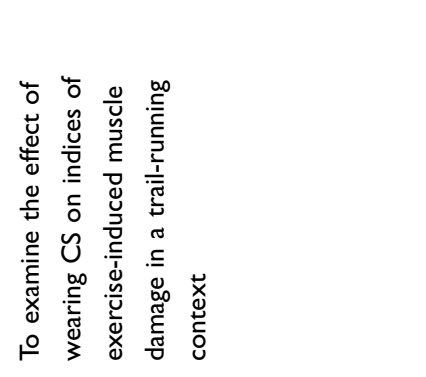 & 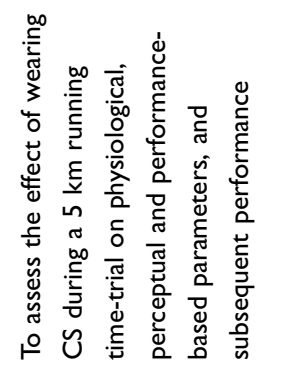 & 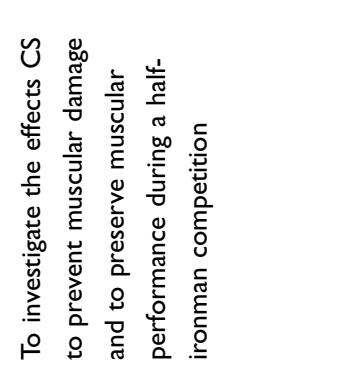 & 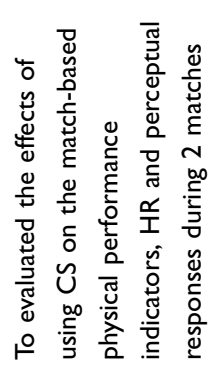 \\
\hline $\begin{array}{l}\infty \\
\stackrel{0}{+} \\
+1 \\
\stackrel{+}{+} \\
\dot{m}\end{array}$ & 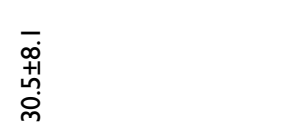 & 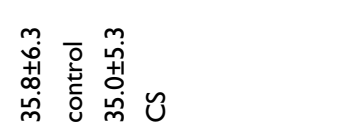 & 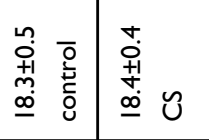 \\
\hline 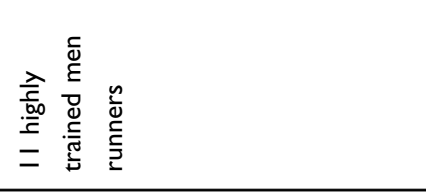 & 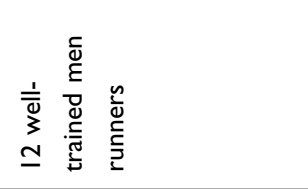 & ల) & 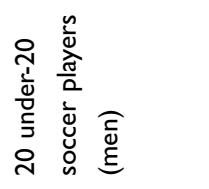 \\
\hline 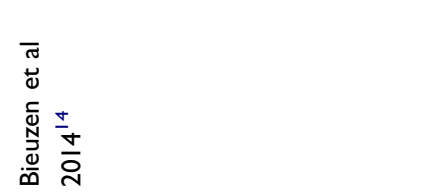 & 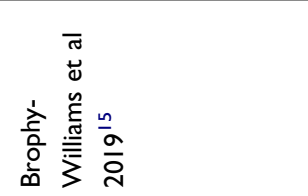 & 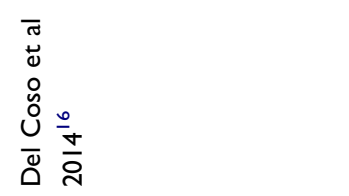 & 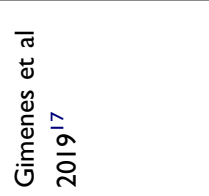 \\
\hline
\end{tabular}




\begin{tabular}{|c|c|c|c|c|c|}
\hline 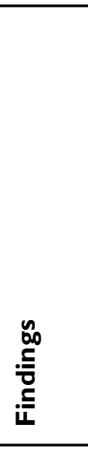 & 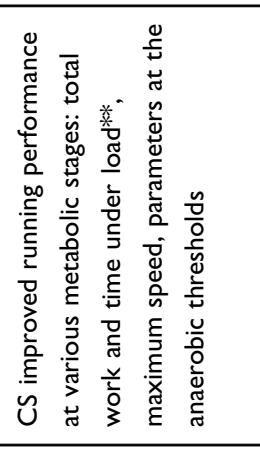 & 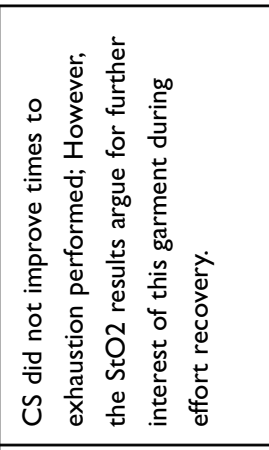 & 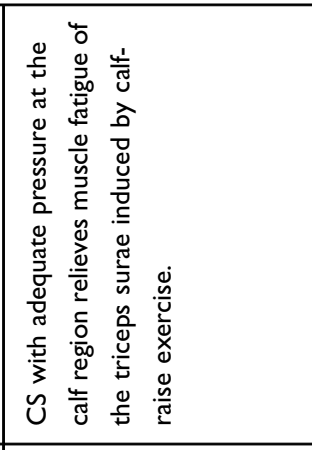 & 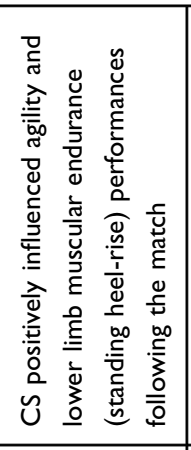 & 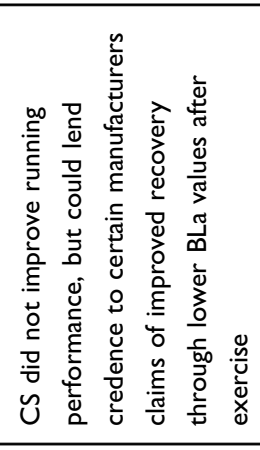 \\
\hline 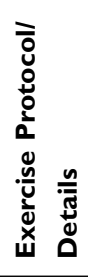 & 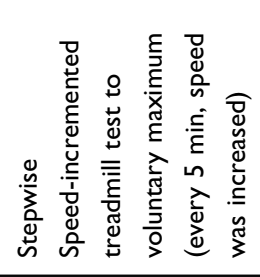 & 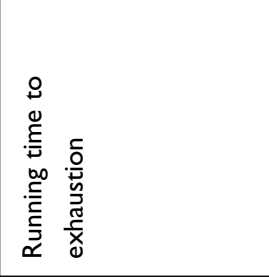 & 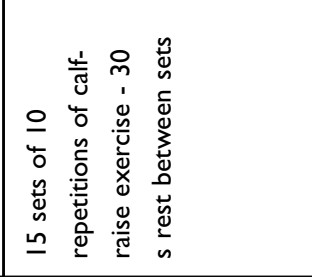 & 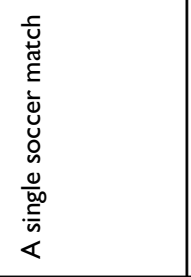 & 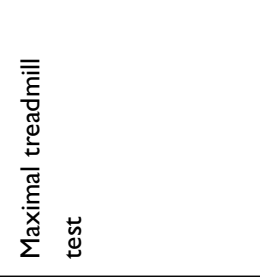 \\
\hline 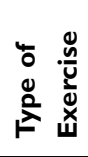 & 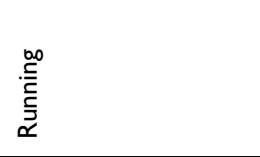 & 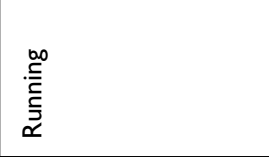 & 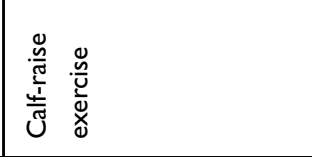 & 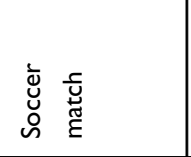 & 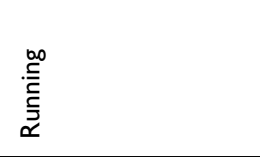 \\
\hline 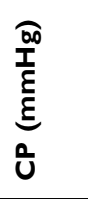 & \pm & 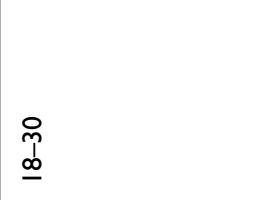 & $\mid \begin{array}{l}0 \\
\text { D } \\
0 \\
\tilde{c} \\
\underline{\infty} \\
\underline{\infty}\end{array}$ & 市 & $\begin{array}{l}\tilde{I} \\
\underline{-1}\end{array}$ \\
\hline 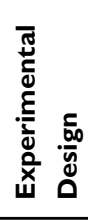 & 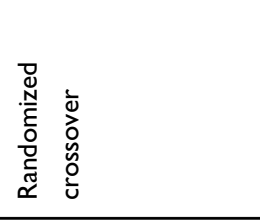 & 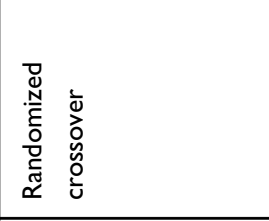 & 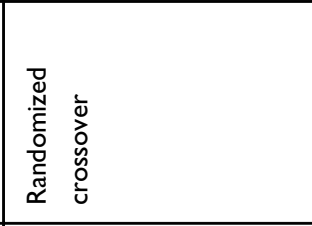 & 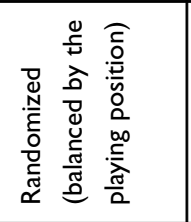 & 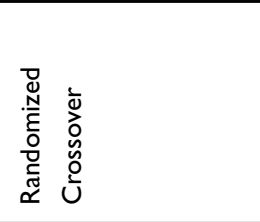 \\
\hline$\frac{E}{4}$ & 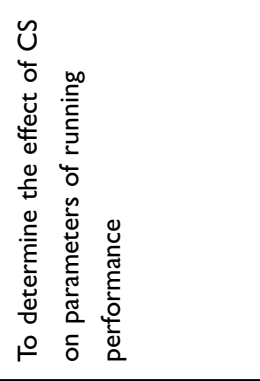 & 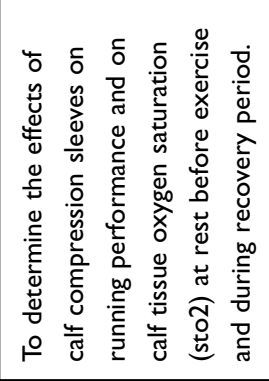 & 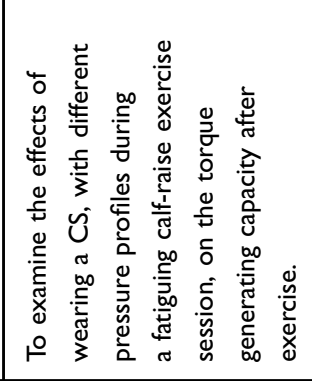 & 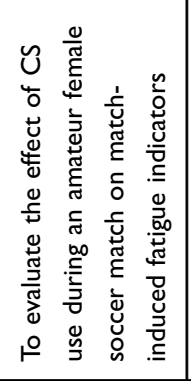 & 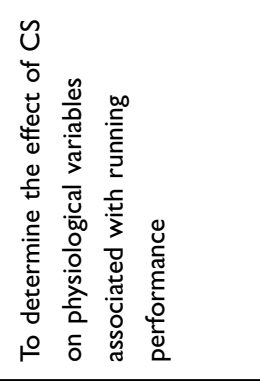 \\
\hline 选 & $\stackrel{m}{\rho} \underset{m}{0} \frac{\hat{o}}{+1}$ & $\begin{array}{l}\hat{i} \\
\dot{1} \\
\dot{N} \\
\dot{N}\end{array}$ & 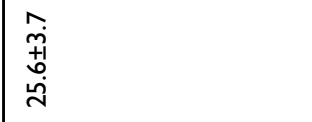 & 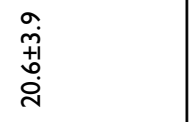 & 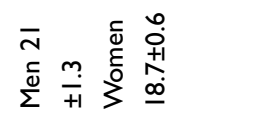 \\
\hline 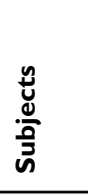 & 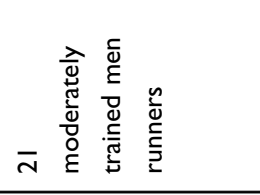 & 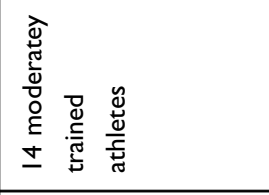 & 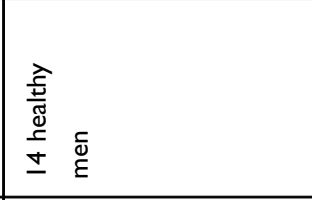 & 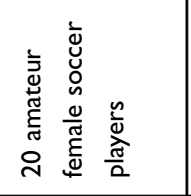 & 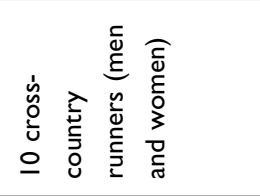 \\
\hline 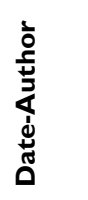 & 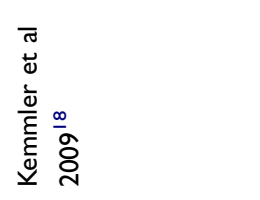 & 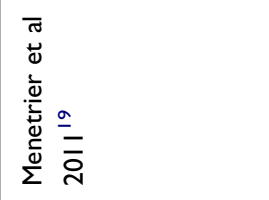 & 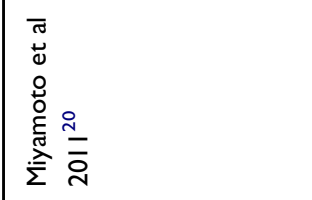 & 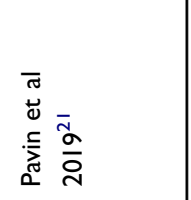 & 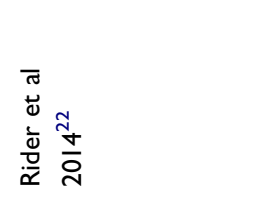 \\
\hline
\end{tabular}




\begin{tabular}{|c|c|c|c|c|}
\hline 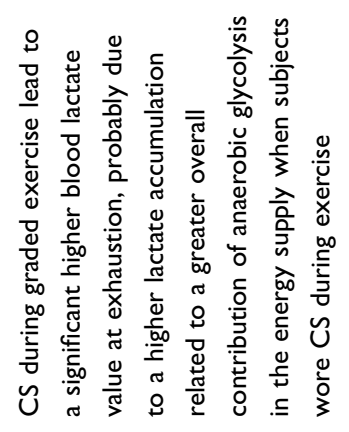 & 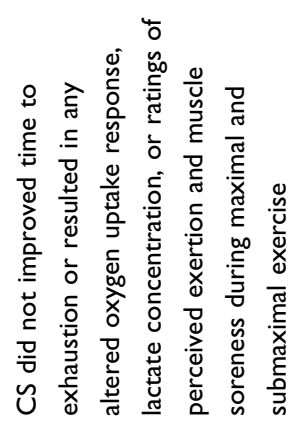 & 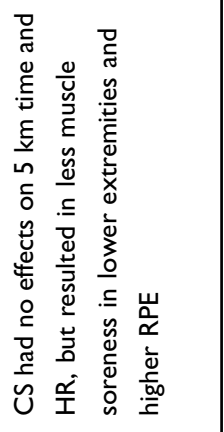 & 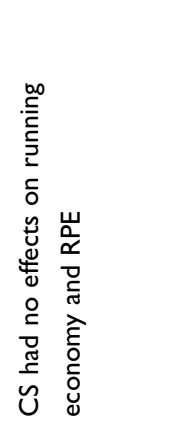 & 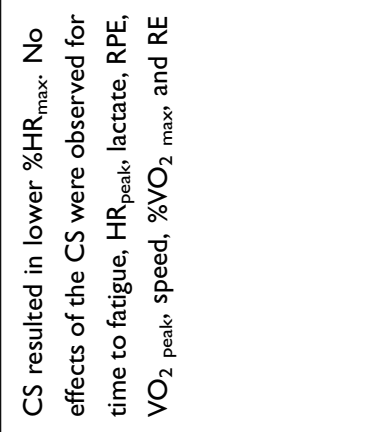 \\
\hline 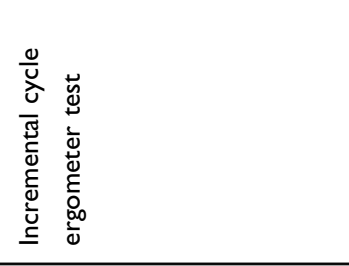 & 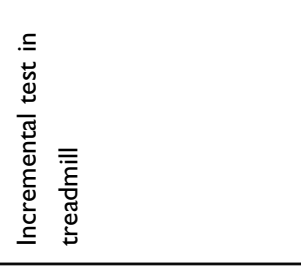 & 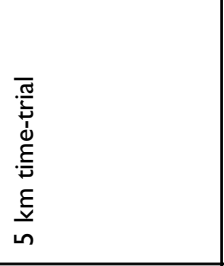 & 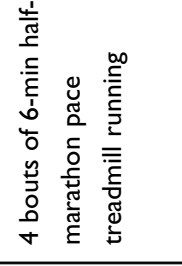 & 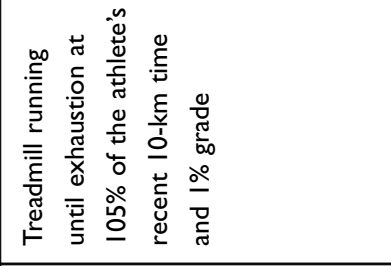 \\
\hline 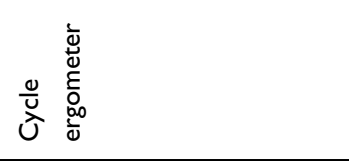 & 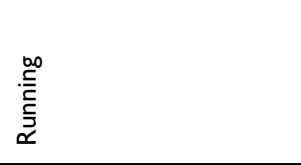 & 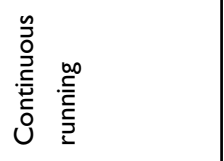 & 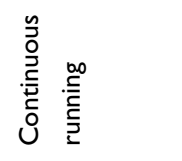 & 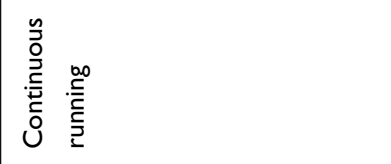 \\
\hline $\begin{array}{l}\underset{I}{I} \\
\stackrel{\text { I }}{2}\end{array}$ & 오 & $\begin{array}{l}\bar{T} \\
\stackrel{d}{ } \\
\underline{d}\end{array}$ & $\begin{array}{l}\tilde{T} \\
\underline{\Lambda}\end{array}$ & $\begin{array}{l}\tilde{N} \\
\underline{\Lambda} \\
\underline{n}\end{array}$ \\
\hline 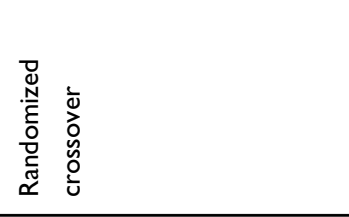 & 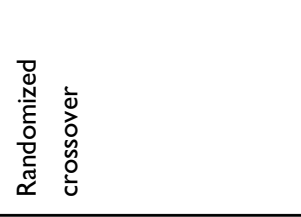 & 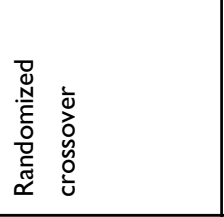 & 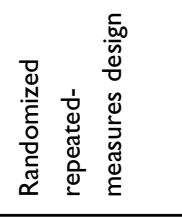 & 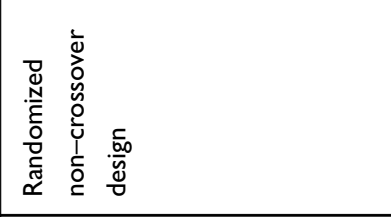 \\
\hline 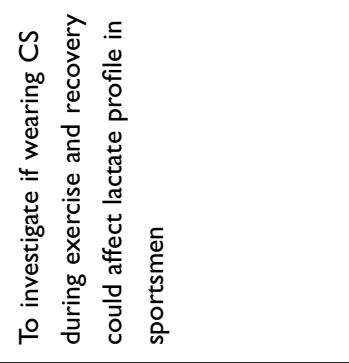 & 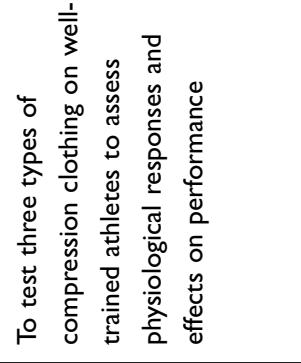 & 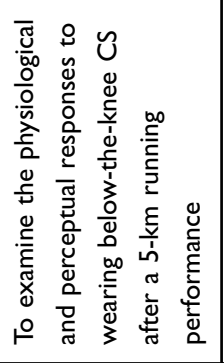 & 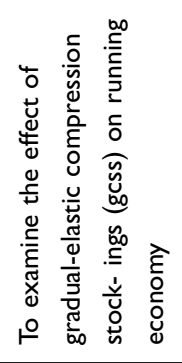 & 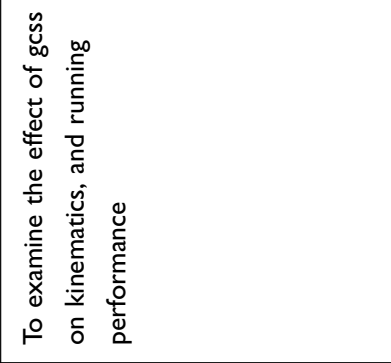 \\
\hline $\begin{array}{l}\stackrel{a}{\dot{c}} \\
\stackrel{+}{+1} \\
\stackrel{N}{N}\end{array}$ & 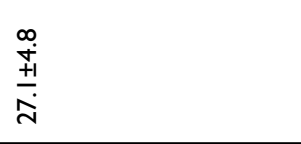 & 華 & $\begin{array}{l}m \\
0 \\
+1 \\
+1 \\
\dot{m}\end{array}$ & 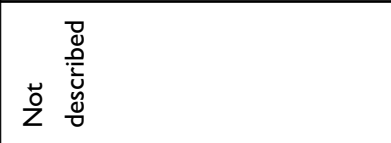 \\
\hline 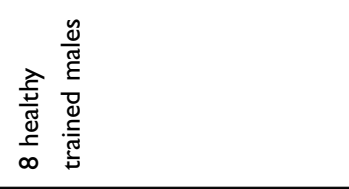 & 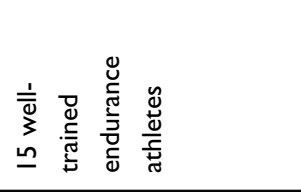 & 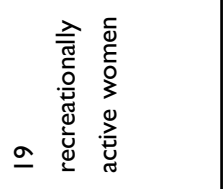 & 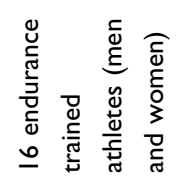 & 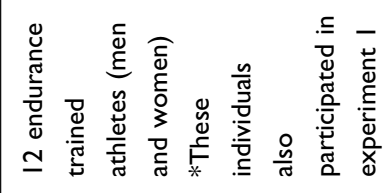 \\
\hline 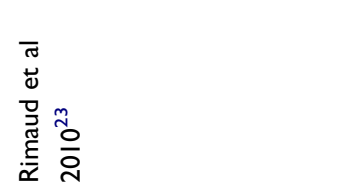 & 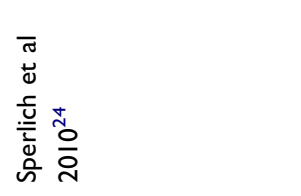 & 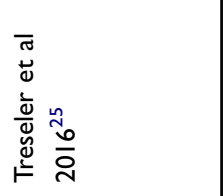 & 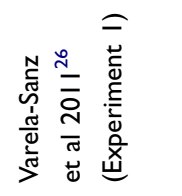 & 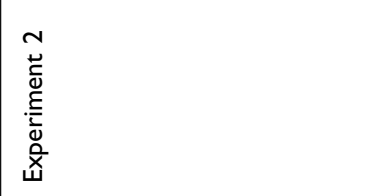 \\
\hline
\end{tabular}




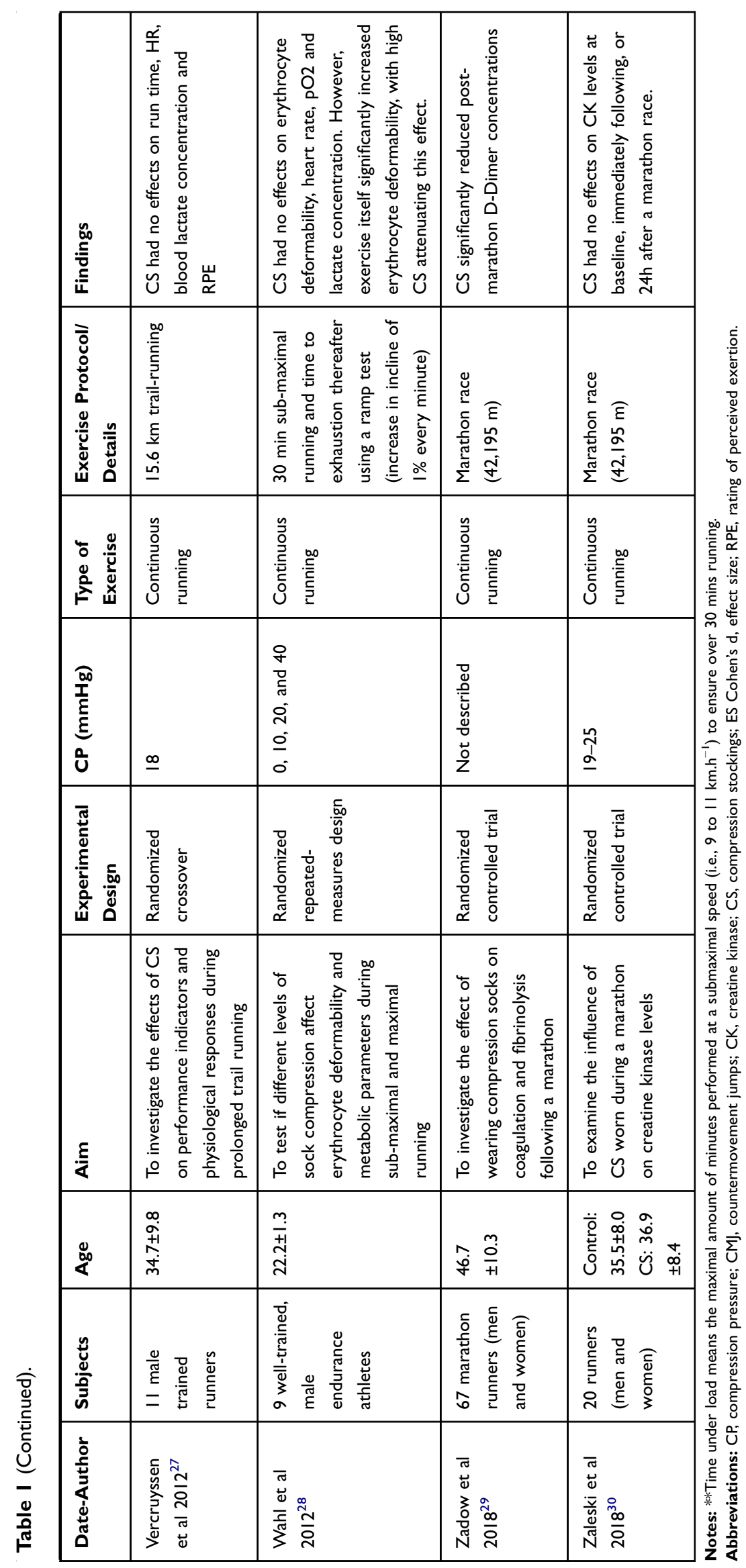


Table 2 Studies That Found Effects from Wearing CS During Exercise

\begin{tabular}{|c|c|c|c|}
\hline $\begin{array}{l}\text { Study Potential } \\
\text { Benefited Variable }\end{array}$ & Summary & Effects from CS & No Effects from CS \\
\hline $\begin{array}{l}\text { Ali et al } 2007^{10} \\
\text { Muscle soreness }\end{array}$ & $\begin{array}{l}\text { Experiment } 2 \text { : CS decreased } \\
\text { muscle soreness following each } \\
\text { exercise bout, and } 24 \mathrm{~h} \text { after the } \\
10 \mathrm{~km} \text { time-trial; } \\
\text { Performance was not influence by } \\
\mathrm{CS}(\mathrm{P}=0.15)\end{array}$ & $\begin{array}{l}\text { Experiment } 2 \text { : } \\
\text { Lower perceived muscle soreness } \\
\text { potential Individual improvements: } \\
10 \text { of the } 14 \text { Participants ran faster } \\
\sim 20 \mathrm{~s}\end{array}$ & $\begin{array}{l}\text { Experiment I: } \\
\text { Distance covered on the multi-stage fitness Shuttle running } \\
\text { test } \\
\text { HRmean } \\
\text { Perceived muscle soreness } \\
\text { RPE } \\
\text { Experiment } 2 \text { : } \\
\text { Time to complete } 10 \mathrm{~km} \text { time-trial (mean) } \\
\text { Time to complete } 1 \mathrm{st} \text { and } 2 \mathrm{nd} 5 \mathrm{~km} \text { partial time RPE }\end{array}$ \\
\hline $\begin{array}{l}\text { Ali et al } 201 \mathrm{I}^{11} \\
\text { Muscle fatigue }\end{array}$ & $\begin{array}{l}\text { CS worn (low and medium } \\
\text { compression) resulted in greater } \\
\text { maintenance of leg power after } \\
10 \mathrm{~km} \text {, but performance on } 10 \mathrm{~km} \\
\text { did not }\end{array}$ & $\begin{array}{l}\text { Vertical jump height higher (from } \\
\text { pre-to post- } 10 \mathrm{~km} \text { running) when } \\
\text { wearing Low }(12-15 \mathrm{~mm} \mathrm{Hg} \text { ) and } \\
\text { Med (I8-2I } \mathrm{mm} \mathrm{Hg}) \mathrm{CS}\end{array}$ & $\begin{array}{l}\text { Time to complete } 10 \mathrm{~km} \\
\text { RPE } \\
\text { HRmean } \\
\text { High }(23-32 \mathrm{~mm} \mathrm{Hg}) \mathrm{CS} \text { had no benefit for vertical jump post- } \\
10 \mathrm{~km}\end{array}$ \\
\hline $\begin{array}{l}\text { Berry et al } 1987^{13} \\
\text { Lactate recovery }\end{array}$ & $\begin{array}{l}\text { CS did not affect the VO2max, } \\
\text { recovery of VO2max, but blood } \\
\text { lactate was lower on the recovery } \\
\text { period when CS was worn during } \\
\text { incremental treadmill test until } \\
\text { exhaustion }\end{array}$ & $\begin{array}{l}\text { Lower blood lactate after the } \\
\text { incremental test (at } 15 \text { min of the } \\
\text { recovery period) }\end{array}$ & $\begin{array}{l}\text { VO2max } \\
\text { Time to exhaustion } \\
\text { recovery of VO2max }\end{array}$ \\
\hline $\begin{array}{l}\text { Bieuzen et al } 2014^{14} \\
\text { Muscle soreness } \\
\text { Muscle fatigue-recovery }\end{array}$ & $\begin{array}{l}\text { CS improved post-exercise } \\
\text { recovery (perceived leg soreness } \\
\text { and muscle function); CS did not } \\
\text { influence the performance } \\
\text { ( } 15.6 \mathrm{~km} \text { in mountainous terrain) } \\
\text { and markers of muscle damage/ } \\
\text { inflammation }\end{array}$ & $\begin{array}{l}\text { Lower perceived muscle soreness } \\
\text { Higher isometric peak torque and } \\
\text { MVC (knee extensors) at I h (ES } \\
\text { small) and } 24 \text { h post-run } \\
\text { All recovery periods on CMJ (ES } \\
\text { large) }\end{array}$ & $\begin{array}{l}\text { Time to complete } 15.6 \mathrm{~km} \\
\text { RPE } \\
\text { HR responses } \\
\text { CK and interleukin- } 6 \text { levels }\end{array}$ \\
\hline $\begin{array}{l}\text { Brophy-Williams et al } \\
2019^{15} \\
\text { Subsequent performance }\end{array}$ & $\begin{array}{l}\text { CS did not affect immediate } \\
\text { performance, but had a positive } \\
\text { impact on subsequent performance } \\
\text { (I h later) }\end{array}$ & $\begin{array}{l}\text { Lower decrement from TTI to } \\
\text { TT2 ( 9.5 s vs control) on time to } \\
\text { complete } 5 \mathrm{~km}\end{array}$ & $\begin{array}{l}\text { Time to complete TTI }(5 \mathrm{~km}) \\
\text { Time to complete TT2 }(5 \mathrm{~km}) \\
\text { Oxygen consumption } \\
\text { Blood lactate } \\
\text { Cross sectional area of calf } \\
\text { RPE } \\
\text { Perceived muscle soreness } \\
\text { Perceived fatigue } \\
\text { Perceived recovery }\end{array}$ \\
\hline $\begin{array}{l}\text { Gimenes et al } 2019^{17} \\
\text { Muscle soreness } \\
\text { Acute performance }\end{array}$ & $\begin{array}{l}\text { CS minimized the increment of } \\
\text { local muscle soreness in the } 2 \text { nd } \\
\text { match (two soccer matches with } \\
72 \mathrm{~h} \text { in-between); CS also } \\
\text { improved performance in high- } \\
\text { intensity activities during the } \\
\text { matches }\end{array}$ & $\begin{array}{l}\text { Minimized the increment of } \\
\text { muscle soreness on match } 2 \text {; } \\
\text { Higher distances covered > } \\
19.1 \mathrm{~km} \cdot \mathrm{h}^{1} \text { and } \geq 23 \mathrm{~km} \cdot \mathrm{h}^{-1} \text { on } \\
\text { match I higher distances covered } \\
\text { between } 19.1 \text { and } 22.99 \mathrm{~km} \cdot \mathrm{h}^{-1} \text { on } \\
\text { match } 2\end{array}$ & $\begin{array}{l}\text { Match I } \\
\text { Perceived soreness and recovery } \\
\text { RPE } \\
\text { HRmean, HRpeak } \\
\text { Internal load (RPE x minutes played) } \\
\text { Sprints repetitions } \\
\text { Distances covered in total and below } 19.1 \mathrm{~km} \cdot \mathrm{h}^{-1} \\
\text { Match } 2 \\
\text { Perceived recovery } \\
\text { RPE } \\
\text { HRmean, HRpeak } \\
\text { Internal load (RPE x minutes played) } \\
\text { Sprints repetitions } \\
\text { Distances covered in total and below } 19.1 \mathrm{~km} \cdot \mathrm{h}^{-1}\end{array}$ \\
\hline
\end{tabular}

(Continued) 
Table 2 (Continued).

\begin{tabular}{|c|c|c|c|}
\hline $\begin{array}{l}\text { Study Potential } \\
\text { Benefited Variable }\end{array}$ & Summary & Effects from CS & No Effects from CS \\
\hline $\begin{array}{l}\text { Kemmler et al } 2009^{18} \\
\text { Acute performance } \\
\text { Anaerobic threshold }\end{array}$ & $\begin{array}{l}\text { CS improved running } \\
\text { performance and metabolic } \\
\text { indicators (anaerobic threshold) }\end{array}$ & $\begin{array}{l}\text { Time under load** (ES } 0.40) \\
\text { Total work (ES: } 0.30) \\
\text { Running at the anaerobic (ES: } \\
0.22 \text { ) } \\
\text { And aerobic thresholds (ES: } 0.28 \text { ) }\end{array}$ & $\begin{array}{l}\text { VO2max } \\
\text { Maximal lactate concentration } \\
\text { HRmax } \\
\text { Pulmonary ventilation } \\
\text { Ventilator equivalent } \\
\text { Respiratory exchange ratio }\end{array}$ \\
\hline $\begin{array}{l}\text { Menetrier et al } 201 I^{19} \\
\text { Oxygen saturation at } \\
\text { recovery }\end{array}$ & $\begin{array}{l}\text { CS did not improve performance, } \\
\text { however CS increased calf tissue } \\
\text { oxygen saturation at rest and } \\
\text { during recovery from exercise }\end{array}$ & $\begin{array}{l}\text { Increased calf tissue oxygen } \\
\text { saturation at rest (before } \\
\text { exercise): }+6.4 \pm 1.9 \% \\
\text { And during recovery: }+7.4 \pm 1.7 \% \\
\text { and }+10.7 \pm 1.8 \% \text { at } 20 \text { th } \\
\text { And } 30 \text { th min of the last recovery } \\
\text { period, respectively }\end{array}$ & $\begin{array}{l}\text { Times to exhaustion performed } \\
\text { HRmean } \\
\text { HRmax } \\
\text { RPE }\end{array}$ \\
\hline $\begin{array}{l}\text { Miyamoto et al } 2011^{20} \\
\text { Muscle fatigue }\end{array}$ & $\begin{array}{l}\text { CS had no effect on the decline of } \\
\text { MVC, but the extent of reduction } \\
\text { of the evoked triplet torque was } \\
\text { smaller when wearing CS with } \\
\text { a high compression pressure }\end{array}$ & $\begin{array}{l}\text { The decline of the MPF in the CS } \\
30 \mathrm{mmHg} \text { was significantly smaller } \\
\text { than that in } 0 \mathrm{mmHg} \text { (control) }\end{array}$ & $\begin{array}{l}\text { Reduction of the MVC torque after the calf-raise among } 0 \\
\text { (control), } 18 \text { and } 30 \mathrm{mmHg} \text { CS } \\
\text { EMG amplitude during the MVC was decreased, the } \\
\text { extent to which was not significantly different among the } \\
\text { three } \\
\text { Conditions both for the medial gastrocnemius and soleus } \\
\text { M-wave amplitude (evoked contraction) }\end{array}$ \\
\hline $\begin{array}{l}\text { Pavin et al } 2019^{21} \\
\text { Muscle fatigue }\end{array}$ & $\begin{array}{l}\text { CS positively influenced agility and } \\
\text { lower limb muscular endurance } \\
\text { performances following a soccer } \\
\text { match }\end{array}$ & $\begin{array}{l}\text { After-match kept the time to } \\
\text { complete } T \text {-test Agility (control } \\
\text { performed slower) from baseline } \\
\text { Control presented greater } \\
\text { decrement after-match (ES }=1.27 \\
\text { control vs. CS) in the heel-rise } \\
\text { test repetitions from baseline }\end{array}$ & $\begin{array}{l}\text { Distance covered in the Yo-Yo intermittent endurance level } \\
2 \text { after match } \\
\text { HRmean, peak and \%peak } \\
\text { RPE }\end{array}$ \\
\hline $\begin{array}{l}\text { Rider et al } 2014^{22} \\
* * \text { worst acute performance } \\
\text { Lactate recovery }\end{array}$ & $\begin{array}{l}\text { CS did not improve running } \\
\text { performance, but seem to } \\
\text { improve recovery after exercise }\end{array}$ & $\begin{array}{l}\text { Time to fatigue lower in CS } \\
(* * \text { negative }) \\
\text { Blood lactate lower during } \\
\text { recovery }(1 \text { and } 5 \mathrm{~min})\end{array}$ & $\begin{array}{l}\text { HR } \\
\text { blood lactate (during the maximal treadmill test) } \\
\text { lactate threshold } \\
\text { VO2max } \\
\text { Respiratory exchange ratio } \\
\text { RPE }\end{array}$ \\
\hline $\begin{array}{l}\text { Rimaud et al } 2010^{23} \\
\text { Lactate recovery }\end{array}$ & $\begin{array}{l}\text { CS did not improve performance } \\
\text { during graded maximal exercise } \\
\text { but lead to a higher contribution } \\
\text { of anaerobic glycolysis and } \\
\text { improved lactate removal during } \\
\text { passive recovery. However, CS } \\
\text { efficacy is highly limited }\end{array}$ & $\begin{array}{l}\text { Higher blood lactate value at } \\
\text { exhaustion } \\
\text { Lactate removal ability was } \\
\text { improved (during passive } \\
\text { recovery) }\end{array}$ & $\begin{array}{l}\text { Submaximal/maximal HR } \\
\text { VO2 } \\
\text { Performance (W on VO2max) } \\
\text { SBP } \\
\text { RPE }\end{array}$ \\
\hline $\begin{array}{l}\text { Treseler et al } 2016^{25} \\
\text { Muscle soreness }\end{array}$ & $\begin{array}{l}\text { CS decreased muscle soreness ( } 24 \\
\text { h post-run) in lower extremities, } \\
\text { (but not for calf) and presented } \\
\text { higher RPE (feelings of working } \\
\text { harder with CS); CS did not } \\
\text { influence } 5 \mathrm{~km} \text { performance } \\
(P=0.74)\end{array}$ & $\begin{array}{l}\text { Lower perceived muscle soreness } \\
24 \mathrm{~h} \text { later } \\
\text { Potential individual improvement } \\
(10 \text { of } 19 \text { participants ran faster } \\
\sim 10 \mathrm{~s})\end{array}$ & $\begin{array}{l}\text { Time to complete } 5 \mathrm{~km} \text { time-trial (mean) } \\
\text { HR responses } \\
\text { Rate of perceived recovery }\end{array}$ \\
\hline
\end{tabular}

(Continued) 
Table 2 (Continued).

\begin{tabular}{|c|c|c|c|}
\hline $\begin{array}{l}\text { Study Potential } \\
\text { Benefited Variable }\end{array}$ & Summary & Effects from CS & No Effects from CS \\
\hline $\begin{array}{l}\text { Varela-Sanz et al } 2011^{26} \\
\text { (experiment 2) } \\
\text { Acute lower cardiac stress }\end{array}$ & $\begin{array}{l}\text { CS resulted in lower cardiac } \\
\text { stress during a test at competition } \\
\text { pace, but none effects for } \\
\text { performance and other } \\
\text { physiological and perceptual } \\
\text { indicators }\end{array}$ & $\begin{array}{l}\text { Lower HR response during a test } \\
\text { at competition pace (ie, 105\% best } \\
10 \mathrm{~km} \text { run) }\end{array}$ & $\begin{array}{l}\text { Time to fatigue } \\
\mathrm{HR}_{\text {peak }} \\
\text { Blood lactate } \\
\mathrm{RPE} \\
\mathrm{VO}_{2 \text { peak }} \text { speed } \\
\% \mathrm{VO}_{2} \text { max } \\
\text { Running economy }\end{array}$ \\
\hline $\begin{array}{l}\text { Zadow et al } 2018^{29} \\
\text { Lower fibrinolytic activity }\end{array}$ & $\begin{array}{l}\text { CS significantly reduced post- } \\
\text { marathon fibrinolytic activity }\end{array}$ & $\begin{array}{l}\text { Lower D-Dimer concentrations } \\
\text { post-marathon }\end{array}$ & $\begin{array}{l}\text { Marathon finishing times } \\
\text { Thrombin-anti-thrombin complex tissue factor } \\
\text { Tissue factor pathway inhibitor }\end{array}$ \\
\hline
\end{tabular}

Notes: **Time under load means the maximal amount of minutes performed at a submaximal speed (ie, 9 to $1 \mathrm{l} \mathrm{km} . \mathrm{h}^{-1}$ ) to ensure over 30 mins running. Abbreviations: CMJ, countermovement jumps; CS, compression stockings; ES Cohen's d, effect size; HR, heart rate; MVC, maximal voluntary contraction; RPE, rating of perceived exertion; TT, time-trial.

Another study showed CS-induced ergogenic effects on performance. The authors found an improvement in running performance concomitantly with anaerobic and aerobic thresholds when participants wore CS. ${ }^{18}$ The benefits of CS-ergogenic effects on performance are attributed to enhanced biomechanical support of the muscles, leading to higher efficiency and lower metabolic costs at given workloads, ${ }^{18,36}$ reduction of muscular microtrauma, ${ }^{6}$ and enhanced the proprioception. ${ }^{32}$ During a $5 \mathrm{~km}$ running time-trial (Brophy-Williams et $\mathrm{al}^{15}$ ) the wearing CS did not affect immediate performance. However, CS generated a positive impact on subsequent $5 \mathrm{~km}$ running (i.e., less performance decrement from time-trial 1 to time-trial 2). Again, the underlying mechanism of such benefit is unclear but may be related to increased oxygen delivery, lower muscle oscillation, and better running mechanics. ${ }^{15}$

Despite the current results, the literature does not indicate robust evidence favoring the use of CS during exercise (i.e., only three studies found benefits on performance). Researchers should be careful in drawing conclusions. Considering that each specific study has (or had) a particular experimental design (e.g., exercise protocol, duration, intensity, variables measured, fitness level of the participants), it becomes difficult to generalize the results from the different studies. Thus, it is essential to consider the risk of bias and heterogeneity of the studies. As the same protocol does not conduct different studies, they will vary in the characteristics of the included population, interventions, diagnostic methods to access outcomes, etc. (clinical heterogeneity). Thus, these studies may be biased. ${ }^{37}$ Additionally, two studies did not find CS-induced effects on group mean performance, but the authors highlighted the individual improvements: 10 of 19 runners ran the $5 \mathrm{~km}$ time-trial approximately $10 \mathrm{~s}$ faster, ${ }^{25}$ and 10 of the 14 runners ran the $10 \mathrm{~km}$ time-trial $^{10}$ approximately $20 \mathrm{~s}$ faster. Therefore, individual responses should be carefully evaluated in practical settings.

\section{CS, Muscle Function and Perceived Muscle Soreness}

Some studies in the current review have shown that CS can induce lower muscle fatigue after an exercise protocol with the same workload than a control condition. ${ }^{11,14,20,21}$ The lower after-exercise fatigue may suggest a preserved muscle function. Overall, such studies show the maintenance (based on baseline values) of muscle function by a smaller decrement of performance (or none) in specific muscular tests performed after the exercise protocol (e.g., running timetrial, soccer match). On the same reasoning, the lower perceived muscle soreness found in the current review is also a potential beneficial outcome from CS. The smaller muscle soreness may be particularly relevant for more prolonged periods with multiples exhausting physical activities performed with a short recovery period in-between. ${ }^{17}$

In one of the studies, competitive runners $\left(\mathrm{VO}_{2} \mathrm{max}\right.$ $\sim 69$ mL.kg.min) completed four $10 \mathrm{~km}$ time-trial wearing control CS $(0 \mathrm{~mm} \mathrm{Hg})$ and CS with different pressures in a randomized, counterbalanced order. ${ }^{11}$ The runners performed CMJ tests before and after running as a muscle function indicator. The results showed that $\mathrm{CMJ}$ height decreased after control running. However, CMJ performance was improved after running wearing CS (low and medium pressure), suggesting a better maintainance of muscle function. 
The authors speculated that improvements in proprioception to jump and reduced muscle oscillations due to CS probably collaborated with lower muscle fatigue. ${ }^{11}$

In other included study, highly trained runners participated in 3 simulated trail races $(15.6 \mathrm{~km}$, including uphill and downhill) in a randomized crossover trial. ${ }^{14}$ Authors measured indicators of muscle function (and also muscle perceived soreness) at baseline, 1, 24, and $48 \mathrm{~h}$ after-run. Muscle function decreased after the race, suggesting the appearance of fatigue, which was partially counteracted by CS. More specifically, a beneficial effect from wearing CS was found for isometric peak torque at $1 \mathrm{~h}$ and $24 \mathrm{~h}$ postrun and for CMJ throughout the $48 \mathrm{~h}$ recovery period. Perceived muscle soreness was also lower when runners wore CS during trail running compared with the control condition ( $1 \mathrm{~h}$ and $24 \mathrm{~h}$ post-run). Specific muscle contractions during trail running (e.g., eccentric on the downhill portion) might result in more extensive muscle oscillation and soreness. Thus, CS probably reduced the perceived muscle soreness due to the higher preservation of muscle function. ${ }^{14}$

Miyamoto et $\mathrm{al}^{20}$ showed that CS promoted a smaller extent of reduction (- $6.4 \pm 8.5 \%$ for CS vs. $-16.5 \pm 9.0 \%$ for control) of the evoked triplet torque, after a fatiguing protocol (15 sets X 10 repetitions) of calf-raise exercise. The authors suggested that mitigation of muscle fatigue observed in their study could be related to increased venous flow velocity and prevention of the lowering of the intramuscular $\mathrm{pH}^{20}$

Positive CS-induced benefits on muscle fatigue was also described after a soccer match. Female players of both teams $(50 \%$ each team, randomly wore CS or control socks) performed tests (agility $\mathrm{T}$, standing heel-rise, and YoYo Intermittent Endurance II) $48 \mathrm{~h}$ before (baseline) and immediately after the game. CS resulted in less matchinduced fatigue for agility $T$-test performance (maintenance for CS and decrement in control players) and heel-rise test (both groups had a decrement on the number of repetitions, but higher in control). ${ }^{21}$

In the current review, some researchers found a beneficial CS-effect on the perceived muscle soreness in lower extremities after the following exercises: highintensity continuous $10 \mathrm{~km}$ road-running, ${ }^{10} 15.6 \mathrm{~km}$ trail in mountainous terrain, ${ }^{14}$ in the second match of soccer ( $72 \mathrm{~h}$ between the first game), ${ }^{17}$ and $24 \mathrm{~h}$ post $5 \mathrm{~km}$ timetrial. ${ }^{25}$ Overall, those studies suggested a lower perception of muscle soreness due to less extensive muscle damage (lower muscle oscillation), and better proprioception.
However, we cannot rule out a potential placebo effect, once it is hard to control such bias due to the nature of compressive CS versus control socks.

\section{CS, Other Potential Benefits, and Final Considerations}

Besides performance, muscle soreness, and muscle function indicators, 15 out of the 21 studies selected in this review presented other variables influenced by CS: lower blood lactate levels, ${ }^{13,22,23}$ and fibrinolytic activity, ${ }^{29}$ higher oxygen saturation, ${ }^{19}$ after the exercise protocol (recovery). Also, lower cardiac stress during exercise has been found. ${ }^{26}$

Mitigation of exercise-induced muscle damage is a possible effect according to authors that found benefit from wearing $\mathrm{CS}$ in this review. However, none of them measured blood markers of muscle damage (e.g., creatine kinase - CK, lactate dehydrogenase - LDH). Curiously, only three studies measured such markers after-exercise: a marathon race, ${ }^{12}$ a $15.6 \mathrm{~km}$ trail-running, ${ }^{14}$ and halfironman triathlon competition, ${ }^{16}$ and found no effect from CS. The lack of measurements of muscle damage markers on several studies herein included may be due to the experimental design and the fact of "only" wearing the CS during the exercise (i.e., more focus on performance than recovery). Longer time-points of measurement after the activity (e.g., time-course of $\mathrm{CK}$ for at least $24 \mathrm{~h}$ after-exercise) could be necessary to detect a significant change in $\mathrm{CK},{ }^{38}$ for example.

Finally, we highlight that in a real-world scenario, athletes probably will not use a promising ergogenic aid to improve performance (e.g., CS) only once, as the majority of studies included here. Athletes would perhaps try it in a couple of training session and one competition before to make a final decision. Also, in practical terms, athletes usually may combine different strategies to improve performance and later recovery, such as ischemic preconditioning, ${ }^{39,40}$ myofascial release, and cold water immersion. ${ }^{41}$ Currently, the effects of such strategies (isolated or combined) with CS are unknown. Therefore, the interpretation of our findings should have in mind "to see also the forest, not just the leaf".

\section{Conclusions}

Wearing below-knee CS during exercise (or sport/physical activity) improved the actual performance in a small number of the studies analyzed. However, there is some evidence that wearing CS could benefit muscle fatigue indicators and muscle soreness immediately after and hours after an exercise 
bout (e.g., better recovery until 48 h). Lower muscle fatigue and muscle soreness might be helpful in subsequent exercises or more extended periods of intervention (e.g., several months). Thus, Sports Medicine professionals should consider the individual responses for performance and a potential placebo (or nocebo) effect. Future studies should evaluate longer experimental designs (e.g., several weeks) wearing CS on exercise performance and physiological indicators, once the chronic effects are unknown.

\section{Disclosure}

The authors report no conflicts of interest in this work.

\section{References}

1. Sigel B, Edelstein AL, Savitch L, Hasty JH, Felix WR Jr. Type of compression for reducing venous stasis. A study of lower extremities during inactive recumbency. Arch Surg. 1975;110(2):171-175. doi:10.1001/archsurg.1975.01360080037005

2. da Silva CA, Helal L, da Silva RP, Belli KC, Umpierre D, Stein R. Association of lower limb compression garments during high-intensity exercise with performance and physiological responses: a systematic review and meta-analysis. Sports Med. 2018;48(8):1859-1873. doi:10.1007/s40279-018-0927-z

3. Gianesini S, Mosti G, Sibilla MG, et al. Lower limb volume in healthy individuals after walking with compression stockings. J Vasc Surg Venous Lymphat Disord. 2019;7(4):557-561. doi:10.1016/j.jvsv.2019. 02.001

4. Gianesini S, Tessari M, Menegatti E, et al. Comparison between the effects of 18 -and $23-\mathrm{mmHg}$ elastic stockings on leg volume and fatigue in golfers. Int Angiol. 2017;36(2):129-135. doi:10.23736/ S0392-9590.16.03647-6

5. Nedelec M, McCall A, Carling C, Legall F, Berthoin S, Dupont G. Recovery in soccer: part I - post-match fatigue and time course of recovery. Sports Med. 2012;42(12):997-1015. doi:10.2165/11635270000000000-00000

6. Valle X, Til L, Drobnic F, et al. Compression garments to prevent delayed onset muscle soreness in soccer players. Muscles Ligaments Tendons J. 2013;3(4):295-302. doi:10.32098/mltj.04.2013.10

7. Priego Quesada JI, Lucas-Cuevas AG, Gil-Calvo M, et al. Effects of graduated compression stockings on skin temperature after running. J Therm Biol. 2015;52:130-136. doi:10.1016/j.jtherbio.2015.06.005

8. Duffield R, Edge J, Merrells R, et al. The effects of compression garments on intermittent exercise performance and recovery on consecutive days. Int J Sports Physiol Perform. 2008;3(4):454-468. doi:10.1123/ijspp.3.4.454

9. Morrison A, Polisena J, Husereau D, et al. The effect of english-language restriction on systematic review-based meta-analyses: a systematic review of empirical studies. Int J Technol Assess Health Care. 2012;28 (2):138-144. doi: $10.1017 / \mathrm{S} 0266462312000086$

10. Ali A, Caine MP, Snow BG. Graduated compression stockings: physiological and perceptual responses during and after exercise. J Sports Sci. 2007;25(4):413-419. doi:10.1080/02640410600718 376

11. Ali A, Creasy RH, Edge JA. The effect of graduated compression stockings on running performance. J Strength Cond Res. 2011;25 (5):1385-1392. doi:10.1519/JSC.0b013e3181d6848e

12. Areces F, Salinero JJ, Abian-Vicen J, et al. The use of compression stockings during a marathon competition to reduce exercise-induced muscle damage: are they really useful? J Orthop Sports Phys Ther. 2015;45(6):462-470. doi:10.2519/jospt.2015.5863
13. Berry MJ, McMurray RG. Effects of graduated compression stockings on blood lactate following an exhaustive bout of exercise. $\mathrm{Am}$ J Phys Med. 1987;66(3):121-132. doi:10.1119/1.18829

14. Bieuzen F, Brisswalter J, Easthope C, Vercruyssen F, Bernard T, Hausswirth C. Effect of wearing compression stockings on recovery after mild exercise-induced muscle damage. Int J Sports Physiol Perform. 2014;9(2):256-264. doi:10.1123/ijspp.2013-0126

15. Brophy-Williams N, Driller MW, Kitic CM, Fell JW, Halson SL. Wearing compression socks during exercise aids subsequent performance. J Sci Med Sport. 2019;22(1):123-127. doi:10.1016/j. jsams.2018.06.010

16. Del Coso J, Areces F, Salinero JJ, et al. Compression stockings do not improve muscular performance during a half-ironman triathlon race. Eur J Appl Physiol. 2014;114(3):587-595. doi:10.1007/s00421013-2789-2

17. Gimenes SV, Marocolo M, Pavin LN, et al. Compression stockings used during two soccer matches improve perceived muscle soreness and high-intensity performance. $J$ Strength Cond Res. 2019. doi:10. 1519/JSC.0000000000003048

18. Kemmler W, von Stengel S, Kockritz C, Mayhew J, Wassermann A, Zapf J. Effect of compression stockings on running performance in men runners. J Strength Cond Res. 2009;23(1):101-105. doi:10.1519/ JSC.0b013e31818eaef3

19. Ménétrier A, Mourot L, Bouhaddi M, Regnard J, Tordi N. Compression sleeves increase tissue oxygen saturation but not running performance. Int J Sports Med. 2011;32(11):864-868. doi:10.1055/ s-0031-1283181

20. Miyamoto N, Hirata K, Mitsukawa N, Yanai T, Kawakami Y. Effect of pressure intensity of graduated elastic compression stocking on muscle fatigue following calf-raise exercise. J Electromyogr Kinesiol. 2011;21(2):249-254. doi:10.1016/j.jelekin.2010.08.006

21. Pavin LN, Leicht AS, Gimenes SV, et al. Can compression stockings reduce the degree of soccer match-induced fatigue in females? Res Sports Med. 2019;27(3):351-364. doi:10.1080/15438627.2018.1527 335

22. Rider BC, Coughlin AM, Hew-Butler TD, Goslin BR. Effect of compression stockings on physiological responses and running performance in division III collegiate cross-country runners during a maximal treadmill test. J Strength Cond Res. 2014;28(6):17 32-1738. doi:10.1519/JSC.0000000000000287

23. Rimaud D, Messonnier L, Castells J, Devillard X, Calmels P. Effects of compression stockings during exercise and recovery on blood lactate kinetics. Eur J Appl Physiol. 2010;110(2):425-433. doi:10.10 07/s00421-010-1503-x

24. Sperlich B, Haegele M, Achtzehn S, Linville J, Holmberg HC, Mester J. Different types of compression clothing do not increase sub-maximal and maximal endurance performance in well-trained athletes. J Sports Sci. 2010;28(6):609-614. doi:10.1080/02640410 903582768

25. Treseler C, Bixby WR, Nepocatych S. The effect of compression stockings on physiological and psychological responses after $5-\mathrm{km}$ performance in recreationally active females. $J$ Strength Cond Res. 2016;30(7):1985-1991. doi:10.1519/JSC.0000000000001291

26. Varela-Sanz A, Espana J, Carr N, Boullosa DA, Esteve-Lanao J. Effects of gradual-elastic compression stockings on running economy, kinematics, and performance in runners. J Strength Cond Res. 2011;25(10):2902-2910. doi:10.1519/JSC.0b013e31820f5049

27. Vercruyssen F, Easthope C, Bernard T, et al. The influence of wearing compression stockings on performance indicators and physiological responses following a prolonged trail running exercise. Eur J Sport Sci. 2014;14(2):144-150. doi:10.1080/ 17461391.2012.730062

28. Wahl P, Bloch W, Mester J, Born DP, Sperlich B. Effects of different levels of compression during sub-maximal and high-intensity exercise on erythrocyte deformability. Eur J Appl Physiol. 2012;112 (6):2163-2169. doi:10.1007/s00421-011-2186-7 
29. Zadow EK, Adams MJ, Wu SSX, et al. Compression socks and the effects on coagulation and fibrinolytic activation during marathon running. Eur J Appl Physiol. 2018;118(10):2171-2177. doi:10.1007/ s00421-018-3929-5

30. Zaleski AL, Pescatello LS, Ballard KD, et al. The influence of compression socks during a marathon on exercise-associated muscle damage. J Sport Rehabil. 2019;28:1-5.

31. Engel FA, Holmberg H-C, Sperlich B. Is there evidence that runners can benefit from wearing compression clothing? Sports Med. 2016;46 (12):1939-1952. doi:10.1007/s40279-016-0546-5

32. Ghai S, Driller MW, Masters RSW. The influence of below-knee compression garments on knee-joint proprioception. Gait Posture. 2018;60:258-261. doi:10.1016/j.gaitpost.2016.08.008

33. Russell M, Sparkes W, Northeast J, et al. Changes in acceleration and deceleration capacity throughout professional soccer match-play. J Strength Cond Res. 2016;30(10):2839-2844. doi:10.1519/JSC.00 00000000000805

34. Doan B, Kwon Y-H, Newton R, et al. Evaluation of a lower-body compression garment. J Sports Sci. 2003;21(8):601-610. doi:10.108 0/0264041031000101971

35. Sperlich B, Born D-P, Swarén M, et al. Is leg compression beneficial for alpine skiers? BMC Sports Sci Med Rehabil. 2013;5(1):18. doi:10.1186/2052-1847-5-18
36. Bringard A, Perrey S, Belluye N. Aerobic energy cost and sensation responses during submaximal running exercise-positive effects of wearing compression tights. Int J Sports Med. 2006;27(5):373-378. doi: $10.1055 / \mathrm{s}-2005-865718$

37. Schulz KF, Grimes DA. Allocation concealment in randomised trials: defending against deciphering. Lancet. 2002;359(9306):614-618. doi:10.1016/S0140-6736(02)07750-4

38. Brancaccio P, Maffulli N, Limongelli FM. Creatine kinase monitoring in sport medicine. Br Med Bull. 2007;81-82:209-230. doi:10. 1093/bmb/ldm014

39. da Mota GR, Willis SJ, Sobral NDS, Borrani F, Billaut F, Millet GP. Ischemic preconditioning maintains performance on two $5-\mathrm{km}$ time trials in hypoxia. Med Sci Sports Exerc. 2019;51(11):2309-2317. doi:10.1249/MSS.0000000000002049

40. Arriel RA, de Souza HLR, da Mota GR, Marocolo M, Zagatto AM. Declines in exercise performance are prevented 24 hours after post-exercise ischemic conditioning in amateur cyclists. PLoS One. 2018;13(11):e0207053. doi:10.1371/journal.pone.0207053

41. Garcia C, da Mota GR, Marocolo M. Cold water immersion is acutely detrimental but increases performance post-12 $\mathrm{h}$ in rugby players. Int J Sports Med. 2016;37(08):619. doi:10.1055/s-00000028

\section{Publish your work in this journal}

Open Access Journal of Sports Medicine is an international, peerreviewed, open access journal publishing original research, reports, reviews and commentaries on all areas of sports medicine. The manuscript management system is completely online and includes a very quick and fair peer-review system. Visit http://www.dovepress. com/testimonials.php to read real quotes from published authors. 